\title{
Lupus-related vasculitis in a cohort of systemic lupus erythematosus patients
}

\author{
Sherif M Gamal ${ }^{1}$, Sally S. Mohamed ${ }^{1}$, Marwa Tantawy ${ }^{2}\left(\mathbb{D}\right.$, Ibrahem Siam $^{3} \mathbb{D}$, \\ Ahmed Soliman ${ }^{4}\left(\mathbb{D}\right.$, Marwa H. Niazy ${ }^{1}(\mathbb{D}$ \\ ${ }^{1}$ Rheumatology, Cairo University, Faculty of Medicine, Cairo, Egypt \\ ${ }^{2}$ Rheumatology, Beni Suif University, Beni Suif, Egypt \\ ${ }^{3}$ Department of Internal Medicine, National Research Centre, Cairo, Egypt \\ ${ }^{4}$ Department of Dermatology, National Research Centre, Cairo, Egypt
}

\begin{abstract}
Objectives: This study aims to examine the frequency and clinical association of lupus-related vasculitis in patients with systemic lupus erythematosus (SLE).

Patients and methods: We retrospectively analyzed medical records of a total of 565 SLE patients (42 males, 523 females; mean age: $32.7 \pm 9.5$ years; range, 13 to 63 years) between January 2017 and February 2020. Demographic, clinical data, and laboratory data and treatment modalities applied were recorded. Lupus-related vasculitis and its different types were documented, and the patients with vasculitis were compared with those without vasculitis.

Results: The mean disease duration was $8.9 \pm 6.3$ years. Vasculitis associated with lupus was found in 191 (33.45\%) patients. Cutaneous vasculitis was found in $59.2 \%$, visceral vasculitis in $34.0 \%$, and both in $6.8 \%$ of total vasculitis patients. The patients with vasculitis had a longer disease duration $(p=0.01)$, were more likely to have juvenile onset $(p=0.002)$, livedo reticularis $(p<0.001)$, Raynaud's phenomenon $(R P)(p<0.001)$, digital gangrene $(p<0.001)$, thrombosis $(p=0.003)$, and cranial neuropathy $(p=0.004)$. The patients with vasculitis showed a higher prevalence of hypercholesterolemia $(p=0.045)$, diabetes mellitus $(p=0.026)$, higher Systemic Lupus Erythematosus Disease Activity Index (SLEDAI) at disease onset $(p<0.001)$, and Systemic Lupus International Collaborating Clinics (SLICC) Damage Index $(p=0.003)$ scores. They had more prevalent hematological manifestations $(p<0.001)$, hypocomplementemia $(p=0.007)$, received a higher cumulative dose of intravenous methylprednisolone $(p<0.001)$, and had also more frequent cyclophosphamide $(p=0.016)$ and azathioprine intake $(p<0.001)$. In the logistic regression analysis, SLE vasculitis was independently associated with juvenile disease onset, livedo reticularis, RP, hematological manifestations, and higher scores of SLEDAl at disease onset $(p<0.05)$.
\end{abstract}

Conclusion: Juvenile disease onset, livedo reticularis, RP, hematological manifestations, and higher SLEDAI scores at disease onset may be associated with the development of vasculitis in SLE patients.

Keywords: Clinical association, frequency, lupus vasculitis.

Systemic lupus erythematosus (SLE) is a multi-systemic autoimmune disease with different forms of clinical characteristics and serological features. ${ }^{1}$ Although the pathogenesis of SLE has not been fully understood, loss of self-tolerance and activation of autoreactive $\mathrm{T}$ and $\mathrm{B}$ cells occurs, leading to the production of pathogenic autoantibodies and tissue injury that characterize the disease. ${ }^{2}$ Vasculitis is inflammation of blood vessel wall, ${ }^{3}$ characterized by infiltration of

Received: February 22, 2021 Accepted: April 19, 2021 Published online: October 12, 2021

Correspondence: Sherif Gamal, MD. Cairo University, Faculty of Medicine, Rheumatology, 11562 Cairo, Egypt. Tel: 00201001811162 e-mail: sherif775@hotmail.com

\section{Citation:}

Gamal S, Mohamed S, Tantawy M, Siam I, Soliman A, Niazy M. Lupus-related vasculitis in a cohort of systemic lupus erythematosus patients. Arch Rheumatol 2021;36(4):595-602. 
inflammatory cells and subsequent necrosis of vessel walls. ${ }^{4}$ The term secondary vasculitis is used to describe inflammation of blood vessels occurring as a complication of underlying disease process (mainly systemic autoimmune diseases as SLE) or triggered by exogenous factors such as infection, drugs, or malignancy. ${ }^{5}$ Lupus vasculitis is classified according to the updated Chapel Hill consensus criteria as vasculitis associated with systemic disease, among secondary vasculitides. ${ }^{6}$ Systemic lupus erythematosus-associated vasculitis may present with different clinical courses, ${ }^{7}$ depending on the site and size of the affected vessels, ${ }^{8}$ the broad spectrum of symptoms and prognosis may range from mild forms, affecting only cutaneous vessels, to severe, catastrophic forms, with organs complications development, and vasculitis within the internal organs which may be life-threatening. ${ }^{7,9}$ Vasculitis can be associated with disease flares in SLE, ${ }^{10,11}$ and may manifest in as high as $56 \%$ of lupus patients throughout their life, with impact on prognosis. The earlier vasculitis is treated, the better is the prognosis of SLE. ${ }^{12}$

On the other hand, there are still limited data about the classification and characteristics of the vasculitides associated with systemic autoimmune diseases as SLE. In the present study, therefore, we aimed to investigate the frequency and clinical association of lupus vasculitis in a cohort of SLE patients.

Table 1. Demographic and clinical characteristics of SLE patients with and without vasculitis

SLE patients $(n=565)$

\begin{tabular}{|c|c|c|c|c|c|c|c|}
\hline \multirow[b]{3}{*}{ Variables } & \multicolumn{6}{|c|}{ SLE patients $(\mathrm{n}=565)$} & \multirow[b]{3}{*}{$p$} \\
\hline & \multicolumn{3}{|c|}{ With lupus vasculitis $(\mathrm{n}=191$ ) } & \multicolumn{3}{|c|}{ Without lupus vasculitis $(n=374)$} & \\
\hline & $\mathrm{n}$ & $\%$ & Mean \pm SD & $\mathrm{n}$ & $\%$ & Mean \pm SD & \\
\hline Age (year) & & & $32.1 \pm 9.3$ & & & $32.9 \pm 9.6$ & 0.3 \\
\hline Sex & & & & & & & 0.69 \\
\hline Female & 178 & 93.2 & & 345 & 92.2 & & \\
\hline Male & 13 & 6.8 & & 29 & 7.8 & & \\
\hline Disease duration (year) & & & $9.8 \pm 6.5$ & & & $8.4 \pm 6.2$ & $0.01^{*}$ \\
\hline Juvenile disease onset & 61 & 31.9 & & 76 & 20.3 & & $0.002^{*}$ \\
\hline Constitutional manifestations & 146 & 76.4 & & 276 & 73.8 & & 0.49 \\
\hline Mucocutaneous manifestations & 176 & 92.1 & & 329 & 88.0 & & 0.13 \\
\hline NPSLE & 87 & 45.5 & & 153 & 40.9 & & 0.29 \\
\hline Cranial neuropathy & 9 & 4.7 & & 3 & 0.8 & & $0.004^{*}$ \\
\hline Peripheral nerve involvement & 14 & 7.3 & & 22 & 5.9 & & 0.51 \\
\hline Nephritis & 126 & 66.0 & & 264 & 70.6 & & 0.26 \\
\hline Renal failure & 15 & 7.9 & & 16 & 4.3 & & 0.08 \\
\hline Arthritis & 178 & 93.2 & & 341 & 91.2 & & 0.41 \\
\hline Cardiovascular manifestations & 56 & 29.3 & & 97 & 25.9 & & 0.39 \\
\hline Pulmonary manifestations & 113 & 59.2 & & 210 & 56.1 & & 0.49 \\
\hline Pulmonary hypertension & 21 & 11.0 & & 38 & 10.2 & & 0.76 \\
\hline Serositis & 108 & 56.5 & & 200 & 53.5 & & 0.49 \\
\hline GIT manifestations & 22 & 11.5 & & 32 & 8.6 & & 0.26 \\
\hline Livedo reticularis & 16 & 8.4 & & 7 & 1.9 & & $<0.001^{*}$ \\
\hline Raynaud's phenomenon & 72 & 37.7 & & 80 & 21.4 & & $<0.001^{*}$ \\
\hline Digital gangrene & 15 & 7.9 & & 4 & 1.1 & & $<0.001^{*}$ \\
\hline Thrombotic events & 43 & 22.5 & & 48 & 12.8 & & $0.003^{*}$ \\
\hline Sicca manifestations & 28 & 14.7 & & 49 & 13.1 & & 0.61 \\
\hline
\end{tabular}




\section{PATIENTS AND METHODS}

This retrospective multi-center study was conducted at Cairo and Beni Suef University Hospital, Department of Rheumatology between January 2017 and February 2020. A total of 565 SLE patients (42 males, 523 females; mean age: $32.7 \pm 9.5$ years; range, 13 to 63 years) who fulfilled the Systemic Lupus International Collaborating Clinics (SLICC) classification criterial $^{13}$ were included. Medical records were reviewed for demographic data, clinical manifestations, routine laboratory investigations and immunological profile including antinuclear antibody (ANA), anti-double stranded deoxyribonucleic acid (anti-DNA), serum complement (C3), and antiphospholipid antibodies (aPL). Also, Systemic Lupus Erythematosus Disease Activity Index (SLEDAI) ${ }^{14}$ was used at the first visit and the last visit for each patient. Accumulated damage was reported according to the SLICC/American College of Rheumatology-Damage Index (ACR-DI). ${ }^{15}$ The details of medical treatment including the total dose of intravenous methylprednisolone and total dose of cyclophosphamide prescribed to the patients since SLE diagnosis, and across all exposure periods until the time of data collection were documented. The study protocol was approved by the National Research Centre Ethics Committee (NRC 20183). The study was conducted in accordance with the principles of the Declaration of Helsinki.

Vasculitic lesions in our series were divided into cutaneous and visceral vasculitis. Similar to Kallas et al.,"16 clinical diagnosis of cutaneous vasculitis was done by an experienced rheumatologist and/or dermatologist (not essentially through histopathology), particularly in typical sites such as palms, soles, fingertips, when a patient presented with palpable purpura or erythematous punctuate lesions in palms, soles, or finger tips not related to palm erythema. Skin lesions related to conditions other than vasculitis such as neoplasms, drug reactions, and SLE-specific cutaneous lesions were excluded. In doubtful cases of cutaneous vasculitis, the definite diagnosis was confirmed by skin biopsy and histopathological reports showing typical findings of lupus-related cutaneous vasculitis. ${ }^{17}$ Visceral vasculitis was confirmed by contrast-enhanced magnetic resonance imaging (MRI) for brain vasculitis, while fundal fluorescein angiography (FFA) was performed for retinal vasculitis, Duplex ultrasound for peripheral arteries, thoracic computed tomography (CT) for intra-alveolar hemorrhage, CT angiography for mesenteric vasculitis, electrocardiography (ECG) and coronary angiography for coronary vasculitis, and nerve biopsy for mono-neuritis multiplex.

Systemic lupus erythematosus-related vasculitis was classified according to the classification of Jennette and Falk ${ }^{3}$ using the names and definitions adopted by the Chapel Hill Consensus Conference. ${ }^{18}$

\section{Statistical analysis}

Statistical analysis was performed using the IBM SPSS version 21.0 software (IBM Corp., Armonk, NY, USA). Quantitative data were expressed in mean \pm standard deviation $(\mathrm{SD})$ for normally distributed data and in median and interquartile range (IQR) for non-normally distributed data. Qualitative data were expressed in number and frequency. The chi-square test was used for testing the association between categorical variables. The Fisher's exact test was used, when the expected frequency count

Table 2. Distribution of vasculitis in lupus patients according to the organs affected

\begin{tabular}{lcc}
\hline Site of vasculitis & \multicolumn{2}{c}{$\begin{array}{c}\text { Secondary } \\
\text { vasculitis lupus } \\
\text { patients ( }=191)\end{array}$} \\
\cline { 2 - 3 } & $\mathrm{n}$ & $\%$ \\
\hline Vasculitis affecting single organ & & \\
Skin vasculitis & 113 & 59.2 \\
Brain vasculitis & 14 & 7.3 \\
Retinal vasculitis & 23 & 12 \\
Peripheral limbs vasculitis (arteritis) & 13 & 6.8 \\
Intra-alveolar hemorrhage & 6 & 3.1 \\
Mesenteric vasculitis & 2 & 1.0 \\
Coronary vasculitis & 1 & 0.5 \\
Mononeuritis mutiplex & 3 & 1.6 \\
Vasculitis affecting more than one organ & & \\
Skin and retinal vasculitis & 8 & 4.2 \\
Skin and brain vasculitis & 1 & 0.5 \\
Skin and limb arteries & 1 & 0.5 \\
Skin and lung & 1 & 0.5 \\
Skin and coronaries & 1 & 0.5 \\
Brain and retinal vasculitis & 2 & 1.0 \\
Brain and lung & 1 & 0.5 \\
Skin, brain and retinal vasculitis & 1 & 0.5 \\
\hline
\end{tabular}


Table 3. Relation between each of comorbidities, disease activity and damage indices, and vasculitis in lupus patients

\begin{tabular}{|c|c|c|c|c|c|c|c|c|c|c|c|}
\hline \multirow[b]{3}{*}{ Variables } & \multicolumn{10}{|c|}{ SLE patients $(\mathrm{n}=565)$} & \multirow[b]{3}{*}{$p$} \\
\hline & \multicolumn{4}{|c|}{ With vasculitis $(n=191)$} & \multirow[b]{2}{*}{ IQR } & \multicolumn{5}{|c|}{ Without vasculitis $(\mathrm{n}=374)$} & \\
\hline & $\mathrm{n}$ & $\%$ & Mean \pm SD & Median & & $\mathrm{n}$ & $\%$ & Mean \pm SD & Median & IQR & \\
\hline Systemic hypertension & 86 & 45.0 & & & & 149 & 39.8 & & & & 0.24 \\
\hline Diabetes mellitus & 24 & 12.6 & & & & 26 & 7.0 & & & & $0.026^{*}$ \\
\hline Dyslipidemia $(\mathrm{n}=531)$ & $89 / 182$ & 48.9 & & & & $161 / 349$ & 46.1 & & & & 0.54 \\
\hline Hypercholesterolemia $(\mathrm{n}=531)$ & $76 / 182$ & 41.8 & & & & $115 / 349$ & 33.0 & & & & $0.045^{*}$ \\
\hline Hypertriglyceridemia $(\mathrm{n}=531)$ & $71 / 182$ & 39.0 & & & & $109 / 349$ & 31.2 & & & & 0.072 \\
\hline Thyroid disease & 17 & 8.9 & & & & 25 & 6.7 & & & & 0.34 \\
\hline AVN & 21 & 11.0 & & & & 28 & 7.5 & & & & 0.16 \\
\hline SLEDAI at onset & & & $16.1 \pm 10.4$ & & & & & $11.8 \pm 7.3$ & & & $<0.001^{*}$ \\
\hline SLEDAI at last visit & & & & 2 & $0-6$ & & & & 4 & $0-8$ & 0.23 \\
\hline SLICC-DI & & & & 1 & $0-2$ & & & & 1 & $0-2$ & $0.002^{*}$ \\
\hline
\end{tabular}

SLE: Systemic lupus erythematosus; SD: Standard deviation; IQR: Interquartile range; AVN: Avascular necrosis; SLEDAI: Systemic Lupus Erythematosus Disease Activity Index; SLICC-DI: Systemic Lupus International Collaborating Clinics-Damage Index; * Significant differences ( $p<0.05)$.

was $<5$. The Student t-test was done to analyze statistically significant differences between the two groups. The Mann-Whitney $\mathrm{U}$ test was performed to compare between non-parametric data of two independent groups. Logistic regression analysis was used to estimate the dependence of SLE secondary vasculitis on a set of independent variables. A $p$ value of $<0.05$ was considered statistically significant.

\section{RESULTS}

The majority of patients were attending Cairo University hospitals during the period from January 2017 until February 2020, while 70 patients were attending Beni Suef University hospitals during the period from January 2019 until February 2020. The mean disease duration was $8.9 \pm 6.3$ (range, 0.5 to 34 ) years. Demographic and clinical

Table 4. Laboratory investigations, immunological profile, and medications in lupus patients with and without vasculitis

\begin{tabular}{|c|c|c|c|c|c|c|c|c|c|c|c|}
\hline \multirow[b]{3}{*}{ Variables } & \multicolumn{10}{|c|}{ SLE patients $(n=565)$} & \multirow[b]{3}{*}{$p$} \\
\hline & \multicolumn{5}{|c|}{ With vasculitis ( $\mathrm{n}=191$ ) } & \multicolumn{5}{|c|}{ Without vasculitis ( $n=374)$} & \\
\hline & $\mathrm{n}$ & $\%$ & Mean \pm SD & Median & IQR & $\mathrm{n}$ & $\%$ & Mean \pm SD & Median & IQR & \\
\hline Hematological manifestations & 187 & 97.9 & & & & 336 & 89.8 & & & & $<0.001^{*}$ \\
\hline $24 \mathrm{~h}$ protein in urine at first (gm/day) & & & & 1 & $0.02-2.7$ & & & & 1.03 & $0-2.24$ & 0.83 \\
\hline Consumed C3 ( $\mathrm{n}=529)$ & $131 / 186$ & 70.4 & & & & $201 / 343$ & 58.6 & & & & $0.007^{*}$ \\
\hline Positive ANA ( $\mathrm{n}=552$ ) & $184 / 188$ & 97.9 & & & & $360 / 364$ & 98.9 & & & & 0.34 \\
\hline Positive anti ds-DNA ( $\mathrm{n}=490$ ) & $127 / 169$ & 75.1 & & & & $222 / 321$ & 69.2 & & & & 0.17 \\
\hline Positive aPL antibodies $(\mathrm{n}=414)$ & $70 / 149$ & 47.0 & & & & $111 / 265$ & 41.9 & & & & 0.32 \\
\hline \multicolumn{12}{|l|}{ Medications received } \\
\hline Intravenous methylprednisolone intake & 177 & 92.7 & & & & 283 & 75.7 & & & & $<0.001^{*}$ \\
\hline $\begin{array}{l}\text { Cumulative intravenous } \\
\text { methyprednisolone dose (g) }\end{array}$ & & & $5.1 \pm 3.6$ & & & & & $3.6 \pm 2.4$ & & & $<0.001^{*}$ \\
\hline Cyclophosphamide intake & 116 & 60.7 & & & & 187 & 50.0 & & & & $0.016^{*}$ \\
\hline Cumulative cyclophosphamide dose (g) & & & $5.8 \pm 3.6$ & & & & & $6.1 \pm 3.3$ & & & 0.37 \\
\hline AZA & 163 & 85.3 & & & & 270 & 72.2 & & & & $<0.001^{*}$ \\
\hline MMF & 57 & 29.8 & & & & 89 & 23.8 & & & & 0.12 \\
\hline Antimalarial drugs intake & 181 & 94.8 & & & & 354 & 94.7 & & & & 0.96 \\
\hline
\end{tabular}

SLE: Systemic lupus erythematosus; SD: Standard deviation; IQR: Interquartile range; C: Complement; ANA: antinuclear antibody; Anti ds-DNA: Anti-double-stranded deoxyribonucleic acid; aPL: Antiphospholipid antibodies; AZA: Azathioprine; MMF: Mycophenolate mofetil; * Significant differences $(\mathrm{p}<0.05)$. 


\begin{tabular}{|c|c|c|c|c|}
\hline Variables & B coefficient & OR & $(95 \% \mathrm{CI})$ for $\mathrm{B}$ & $p$ \\
\hline Disease duration & 0.001 & 1.001 & $0.966-1.036$ & 0.977 \\
\hline Juvenile disease onset & 0.524 & 1.688 & $1.032-2.761$ & $0.037^{*}$ \\
\hline Livedo reticularis & 1.449 & 4.259 & $1.186-15.289$ & $0.026^{*}$ \\
\hline Raynaud's phenomenon & 0.504 & 1.655 & $1.044-2.624$ & $0.032^{*}$ \\
\hline Hematological manifestations & 1.371 & 3.938 & $1.294-11.982$ & $0.016^{*}$ \\
\hline SLEDAI at disease onset & 0.052 & 1.053 & $1.028-1.080$ & $<0.001^{*}$ \\
\hline SLICC-DI & 0.074 & 1.007 & $0.947-1.224$ & 0.258 \\
\hline Hypocomplementemia & 0.28 & 1.323 & $0.842-2.078$ & 0.225 \\
\hline Hypercholesterolemia & -0.007 & 0.993 & $0.639-1.544$ & 0.976 \\
\hline Diabetes mellitus & 0.424 & 1.528 & $0.765-3.054$ & 0.23 \\
\hline Constant & -3.18 & 0.042 & - & - \\
\hline
\end{tabular}

data of the patients with and without vasculitis are shown in Table 1.

Lupus-related vasculitis was found in 191 (33.45\%) of our patients, cutaneous vasculitis in $113(59.2 \%)$ SLE patients, visceral vasculitis in 65 (34.0\%) lupus patients, and combined cutaneous and visceral in $13(6.8 \%)$ patients. The body organs involved in patients with vasculitis are shown in Table 2 .

Comparison between the patients with and without vasculitis in our cohort regarding comorbidities (SLEDAI-SLICC) showed that total cholesterol, the estimated SLEDAI at disease onset, and SLICC scores were statistically significantly higher in the group of vasculitis. However, there was no statistically significant difference between the two groups regarding the SLEDAI scores at the last visit (Table 3). Mortality rate was significantly higher in the vasculitis group: the mortality rate in patients with vasculitis was $12 \%(23 / 191)$ versus $3.7 \%(14 / 374)$ in patients without $(\mathrm{p}<0.001)$. Also, comparison of both groups regarding laboratory findings showed that the vasculitis group had a statistically significant difference regarding hematological

\begin{tabular}{|c|c|c|c|c|}
\hline Variables & B coefficient & OR & $(95 \% \mathrm{CI})$ for $\mathrm{B}$ & $p$ \\
\hline Age & -0.027 & 0.973 & $0.944-1.004$ & 0.085 \\
\hline Sex & 0.383 & 1.466 & $0.412-5.216$ & 0.554 \\
\hline NPSLE & 1.08 & 2.945 & $1.697-5.111$ & $<0.001^{*}$ \\
\hline Renal failure & -0.923 & 0.397 & $0.087-1.805$ & 0.232 \\
\hline Digital gangrene & 2.348 & 10.462 & $3.275-33.420$ & $<0.001^{*}$ \\
\hline Thrombosis & 0.803 & 2.232 & $1.183-4.210$ & $0.013^{*}$ \\
\hline Thrombocytopenia & 0.606 & 1.832 & $1.056-3.179$ & $0.031^{*}$ \\
\hline SLEDAI at disease onset & 0.055 & 1.056 & $1.028-1.085$ & $<0.001^{*}$ \\
\hline Constant & -3.083 & 0.046 & - & - \\
\hline
\end{tabular}


manifestations and C3 consumption, compared to the other group.

The medications used are shown in Table 4. Lupus patients with vasculitis tended to receive higher cumulative doses of intravenous methylprednisolone and to have a higher frequency of cyclophosphamide and azathioprine intake compared to the other group, indicating a statistically significant difference ( $p=0.016, p<0.001$, respectively).

In the logistic regression analysis, vasculitis in SLE was significantly associated with juvenile onset of lupus disease, livedo reticularis, Raynaud's phenomenon, hematological manifestations, and higher scores of SLEDAI at the onset of the disease (Table 5). On analyzing associated clinical features and laboratory investigations by logistic regression, the visceral vasculitis was significantly associated with neuropsychiatric SLE (NPSLE), digital gangrene, thrombosis, and thrombocytopenia (Table 6).

\section{DISCUSSION}

Vascular involvement is common in SLE patients and is implicated as one of the most frequent causes of mortality in established disease. ${ }^{19}$ Vasculitis in SLE is among the most typical processes involved in the clinical evolution of the disease. ${ }^{20}$ The presence of vasculitis appears to be an important turning point in disease process in SLE, ${ }^{7}$ as it can adversely affect the disease outcome and change therapeutic decisions.

In the current study, SLE-related vasculitis was found in $33.5 \%$. Similarly, the frequency of vasculitis in SLE patients ranges from 11 to $36 \%$ in the literature. $.^{4,5}, 21$ It has been also reported that about 21 to $70 \%$ of SLE patients may develop systemic vasculitis at some point of their illness. ${ }^{22}$ In the present study, the patients with vasculitis tended to have a longer disease duration and juvenile onset compared to those without vasculitis, consistent with previous results. ${ }^{21,23}$ Females represented $93.2 \%$ among lupus vasculitis cases, which is compatible with the Shahin et al.'s ${ }^{24}$ study reporting as $94.6 \%$ females among vasculitis cases secondary to SLE. Previous studies reported that cutaneous vasculitis was the most predominant type of vasculitis in SLE patients occurring up to six to eight times more frequently than visceral vasculitis. ${ }^{5,21}$ Likewise, our study showed that cutaneous vasculitis was more common (1.7-fold) than visceral vasculitis. The higher percentage of visceral vasculitis in our study may be due to the inclusion of retinal vasculitis which was present in $12 \%$ of patients. Retinal involvement in SLE patients has been described in 7 to $26 \%$ of cases in the literature. ${ }^{25}$

In the present study, similar to previous studies, a significant association of vasculitis with clinical manifestations as livedo reticularis, ${ }^{5,21}$ and Raynaud's phenomenon ${ }^{21,26}$ was found. Digital gangrene and thrombotic events were significantly higher in our lupus vasculitis cohort. Supportively, Crowson and Magro ${ }^{27}$ reported that digital infarctions suggested underlying vasculitis, unless they were a result of Raynaud's phenomenon. Emmi et al. ${ }^{28}$ showed that, although inflammationinduced thrombosis was considered a feature of autoimmune diseases as SLE, it also played a role in systemic vasculitis. Cranial neuropathy was found to be significantly associated with vasculitis group in our study; however, the limited number of data in the literature and a few number of patients with cranial neuropathy in our study suggest that further studies are needed to confirm this finding.

It is worth noting that patients with lupus vasculitis in our cohort had a significantly higher total cholesterol levels and a higher frequency of diabetes mellitus (DM) compared to the other group. It is well known that patients with DM are significantly at an increased risk for vascular complications with changes in cellular homeostasis and regulation of vascular physiology which, in turn, have an impact on the major functions of vascular cells. ${ }^{29,30}$ Additionally, dyslipidemia has a definite effect on cardiovascular disease and subclinical atherosclerosis. Synergistically, DM and dyslipidemia contribute to increased vascular morbidity in autoimmune diseases as SLE. ${ }^{31}$

A higher frequency of hypocomplementemia in the vasculitis group was elicited in the present study, coinciding with the previously reported studies. ${ }^{5,7,22}$ The presence of hypocomplementemia has been explained 
as a part of disease pathogenesis involving immune complex deposition in the vessel wall. ${ }^{32}$ Although, as previously described, many clinical and laboratory parameters were significantly associated with vasculitis, logistic regression analysis showed that the main association of lupus vasculitis in our cohort was juvenile onset of lupus disease, livedo reticularis, Raynaud's phenomenon, hematological manifestations, and higher scores of SLEDAI at the onset of the disease. Drenkard et al. ${ }^{21}$ reported an association of Raynaud's phenomenon with lupus vasculitis in SLE in the univariate and multivariate analyses. A higher incidence of hematological manifestations as anemia, ${ }^{5}$ and leucopenia ${ }^{21,22}$ in the group with vasculitis was elicited in the aforementioned studies, as well as in the present study. Visceral vasculitis in SLE patients was significantly associated with NPSLE, digital gangrene, thrombosis and thrombocytopenia, and higher scores of SLEDAI at the onset of disease. NPSLE may occur as a result of impaired vascular supply due to vasculitis of cerebral vessels and increased risk of thrombosis.

In the current study, the vasculitis group tended to receive a higher cumulative dose of intravenous methylprednisolone, as well as a higher intake of cyclophosphamide and azathioprine. Indeed, this could be expected, as lupus vasculitis tends to occur more commonly during the disease flares. ${ }^{5,7,21}$ This is supported by the fact that vasculitis is a well-recognized item of several disease activity assessment tools, including the SLEDAI. ${ }^{14}$

Nonetheless, there are some limitations to this study. Biopsy was unavailable for all cutaneous vasculitis patients. Full aPL profile was unable to be documented in some medical records of our patients, also anti-Ro and anti-La antibodies were excluded from our analysis, as they were missing in more than $25 \%$ of our medical records. Therefore, we recommend that further studies should be conducted to investigate the role of these autoantibodies as predictors of lupus vasculitis.

In conclusion, juvenile onset lupus, longer disease duration, livedo reticularis, Raynaud's phenomenon, hematological manifestations, and higher SLEDAI scores at the onset of the disease are associated with lupus vasculitis. Nevertheless, further studies are warranted to confirm whether they have an association or represent risk factors for the development of lupus vasculitis.

\section{Declaration of conflicting interests}

The authors declared no conflicts of interest with respect to the authorship and/or publication of this article.

\section{Funding}

The authors received no financial support for the research and/or authorship of this article.

\section{REFERENCES}

1. Fukuda MV, Lo SC, de Almeida CS, Shinjo SK. AntiRo antibody and cutaneous vasculitis in systemic lupus erythematosus. Clin Rheumatol 2009;28:301-4.

2. Albirdisi MR, Al-Homood IA. Characteristics of lupus nephritis in Saudi lupus patients: A retrospective observational study. Lupus 2020;29:1638-43.

3. Jennette JC, Falk RJ. Small-vessel vasculitis. N Engl J Med 1997;337:1512-23.

4. Barile-Fabris L, Hernández-Cabrera MF, BarraganGarfias JA. Vasculitis in systemic lupus erythematosus. Curr Rheumatol Rep 2014;16:440.

5. Ramos-Casals M, Nardi N, Lagrutta M, BritoZerón P, Bové A, Delgado G, et al. Vasculitis in systemic lupus erythematosus: Prevalence and clinical characteristics in 670 patients. Medicine (Baltimore) 2006;85:95-104.

6. Jennette JC, Falk RJ, Bacon PA, Basu N, Cid MC, Ferrario F, et al. 2012 revised International Chapel Hill Consensus Conference Nomenclature of Vasculitides. Arthritis Rheum 2013;65:1-11.

7. Gheita TA, Abaza NM, Sayed S, El-Azkalany GS, Fishawy HS, Eissa AH. Cutaneous vasculitis in systemic lupus erythematosus patients: Potential key players and implications. Lupus 2018;27:738-43.

8. Calamia KT, Balabanova M. Vasculitis in systemic lupus erythematosis. Clin Dermatol 2004;22:148-56.

9. Saleh A, Stone JH. Classification and diagnostic criteria in systemic vasculitis. Best Pract Res Clin Rheumatol 2005;19:209-21.

10. Petri MA, van Vollenhoven RF, Buyon J, Levy RA, Navarra SV, Cervera R, et al. Baseline predictors of systemic lupus erythematosus flares: Data from the combined placebo groups in the phase III belimumab trials. Arthritis Rheum 2013;65:2143-53.

11. Smith EMD, Lythgoe H, Hedrich CM. Vasculitis in juvenile-onset systemic lupus erythematosus. Front Pediatr 2019;7:149.

12. Pyrpasopoulou A, Chatzimichailidou S, Aslanidis S. Vascular disease in systemic lupus erythematosus. Autoimmune Dis 2012;2012:876456. 
13. Petri M, Orbai AM, Alarcón GS, Gordon C, Merrill JT, Fortin PR, et al. Derivation and validation of the Systemic Lupus International Collaborating Clinics classification criteria for systemic lupus erythematosus. Arthritis Rheum 2012;64:2677-86.

14. Bombardier C, Gladman DD, Urowitz MB, Caron D, Chang $\mathrm{CH}$. Derivation of the SLEDAI. A disease activity index for lupus patients. The Committee on Prognosis Studies in SLE. Arthritis Rheum 1992;35:630-40.

15. Gladman D, Ginzler E, Goldsmith C, Fortin P, Liang M, Urowitz $\mathrm{M}$, et al. The development and initial validation of the Systemic Lupus International Collaborating Clinics/American College of Rheumatology damage index for systemic lupus erythematosus. Arthritis Rheum 1996;39:363-9.

16. Kallas R, Goldman D, Petri MA. Cutaneous vasculitis in SLE. Lupus Sci Med 2020;7:e000411.

17. Carlson JA. The histological assessment of cutaneous vasculitis. Histopathology 2010;56:3-23.

18. Jennette JC, Falk RJ, Andrassy K, Bacon PA, Churg J, Gross WL, et al. Nomenclature of systemic vasculitides. Proposal of an international consensus conference. Arthritis Rheum 1994;37:187-92.

19. Radic M, Martinovic Kaliterna D, Radic J. Vascular manifestations of systemic lupus erythematosis. Neth J Med 2013;71:10-6.

20. Doyle MK. Vasculitis associated with connective tissue disorders. Curr Rheumatol Rep 2006;8:312-6.

21. Drenkard C, Villa AR, Reyes E, Abello M, AlarcónSegovia D. Vasculitis in systemic lupus erythematosus. Lupus 1997;6:235-42.

22. Toubi E, Kessel A, Bamberger E, Golan TD. Systemic lupus erythematosus vasculitis: A current therapeutic overview. Curr Treat Options Cardiovasc Med 2004;6:87-97.
23. Uthman I. Pharmacological therapy of vasculitis: An update. Curr Opin Pharmacol 2004;4:177-82.

24. Shahin AA, Zayed HS, Elrefai RM, Taher H, Elsaie $\mathrm{A}$, Senara $\mathrm{SH}$, et al. The distribution and outcome of vasculitic syndromes among Egyptians: A multicentre study including 630 patients. The Egyptian Rheumatologist 2018;40:243-8.

25. Read RW. Clinical mini-review: Systemic lupus erythematosus and the eye. Ocul Immunol Inflamm 2004; 12:87-99.

26. Shinjo SK, Bonfá E. Cutaneous vasculitis in systemic lupus erythematosus: Association with anti-ribosomal $\mathrm{P}$ protein antibody and Raynaud phenomenon. Clin Rheumatol 2011;30:173-7.

27. Crowson AN, Magro CM. Cutaneous histopathology of lupus erythematosus. Diagnostic Histopathology 2009; 15:157-85.

28. Emmi G, Silvestri E, Squatrito D, Amedei A, Niccolai E, D'Elios MM, et al. Thrombosis in vasculitis: From pathogenesis to treatment. Thromb J 2015;13:15.

29. Rask-Madsen C, King GL. Vascular complications of diabetes: Mechanisms of injury and protective factors. Cell Metab 2013;17:20-33.

30. Gregg EW, Li Y, Wang J, Burrows NR, Ali MK, Rolka $\mathrm{D}$, et al. Changes in diabetes-related complications in the United States, 1990-2010. N Engl J Med 2014;370:1514-23.

31. Tselios K, Koumaras C, Gladman DD, Urowitz MB. Dyslipidemia in systemic lupus erythematosus: Just another comorbidity? Semin Arthritis Rheum 2016;45:604-10.

32. Cuellar ML, Espinoza LR. Laboratory testing in the evaluation and diagnosis of vasculitis. Curr Rheumatol Rep 2000;2:417-22. 